\title{
Model of the RNA Polymerase Complex of the SARS-CoV-2 Virus with Favipiravir
}

\author{
I. V. Polyakov ${ }^{a, b}$, B. L. Grigorenko ${ }^{a, b}$, and A. V. Nemukhin ${ }^{a, b}, *$ \\ ${ }^{a}$ Emanuel Institute of Biochemical Physics, Russian Academy of Sciences, Moscow, 119991 Russia \\ ${ }^{b}$ Moscow State University, Moscow, 119991 Russia \\ *e-mail: anemukhin@yahoo.com
}

Received July 2, 2020; revised July 27, 2020; accepted September 21, 2020

\begin{abstract}
A model of a multidomain complex is constructed using molecular modeling methods to explain the mechanism of the inhibitory effect of favipiravir on RNA-dependent RNA polymerase $(R d R p)$ of the SARS-CoV-2 coronavirus. As the initial atomic coordinates, we use cryoelectron microscopy data for the apo form of RdRp of the SARS-CoV-2 virus and data on the structure of RdRp of the hepatitis $C$ virus. After appropriate substitutions, an RdRp complex containing RNA chains and a potential enzyme inhibitor, favipiravir in the form of ribosatriphosphate, are constructed. The structure of the complex in aqueous shells, which includes more than 100000 atoms, is optimized by molecular dynamics methods. Analysis of the active site with the incorporated favipiravir molecule makes it possible to explain the chemical reaction of the enzyme with the inhibitor.
\end{abstract}

Keywords: molecular dynamics, coronavirus, RNA polymerase, reaction mechanism, inhibition, favipiravir

DOI: $10.1134 /$ S1990793121010255

\section{INTRODUCTION}

The COVID-19 coronavirus pandemic has stimulated research on the molecular processes occurring in all components of the SARS-CoV-2 virus, including its most important part, RNA-dependent RNA polymerase (RdRp), which is responsible for the replication and transcription of genetic information [1]. Potential RdRp inhibitors are considered promising drugs for the treatment of the disease, and favipiravir is one of the most interesting candidates [2]. It should be noted that at the time of writing, the corresponding drug was approved by the Ministry of Health of the Russian Federation for the treatment of COVID-19.

Figure 1 schematically shows the mechanism of chemical transformations in the active site of RNA polymerase. Upon the deprotonation of ribose $\mathrm{O}^{3 \prime}$ of the terminal nucleotide in the $\mathrm{NB}(i)$ (left part of Fig. 1), a nucleophilic attack occurs of $\mathrm{O}^{3 \prime}$ on the $\mathrm{P}^{\mathrm{A}}$ atom of the free nucleotide $\mathrm{NB}(i+1)$ (central part of Fig. 1), which leads to the breaking of the $\mathrm{P}^{\mathrm{A}}-\mathrm{O}^{3 \mathrm{~A}}$ bond, cleavage of pyrophosphate, and extension of the RNA chain (right side of Fig. 1). The amino acid residue of aspartic acid can act as a generalized base (B) at the deprotonation step. The active center contains metal ions $\mathrm{M}_{\mathrm{A}}^{2+}$, and $\mathrm{M}_{\mathrm{B}}^{2+}$, where $\mathrm{M}$ is magnesium or manganese coordinating negatively charged groups of reactants.
Disruption of the functioning of this molecular machine leads to the blocking of the multiplication of the virus. Figure 2 shows a molecule of favipiravir (in conjunction with ribosatriphosphate (FRTP)), which can compete with the native substrates of the enzyme in the active site and stop unwanted synthesis of the RNA virus.

This paper aims to study the physicochemical aspects of biomolecular processes related to the inhibition of the most important enzyme RdRp of the SARS-CoV-2 virus. We use molecular modeling tools to construct and analyze the active site of RNA polymerase including the favipiravir molecule. The cryoelectron microscopy data obtained in 2020 for the apo-form RdRp [3], as well as the known results on the crystal structure of RdRp of the hepatitis $C$ virus [4], provide the initial structural information based on which it is possible to extend the modeling to the analysis of molecular groups in the enzyme's configuration with a cofactor (favipiravir in the form of ribosatriphosphate), imitating an enzyme-substrate complex. Information about the mechanism of the chemical reaction in RNA polymerase [5] and the experience of modeling enzyme-catalyzed reactions of nucleoside triphosphate conversion by quantum mechanics/molecular mechanics [6, 7] allow us to explain the inhibitory effect of favipiravir. 


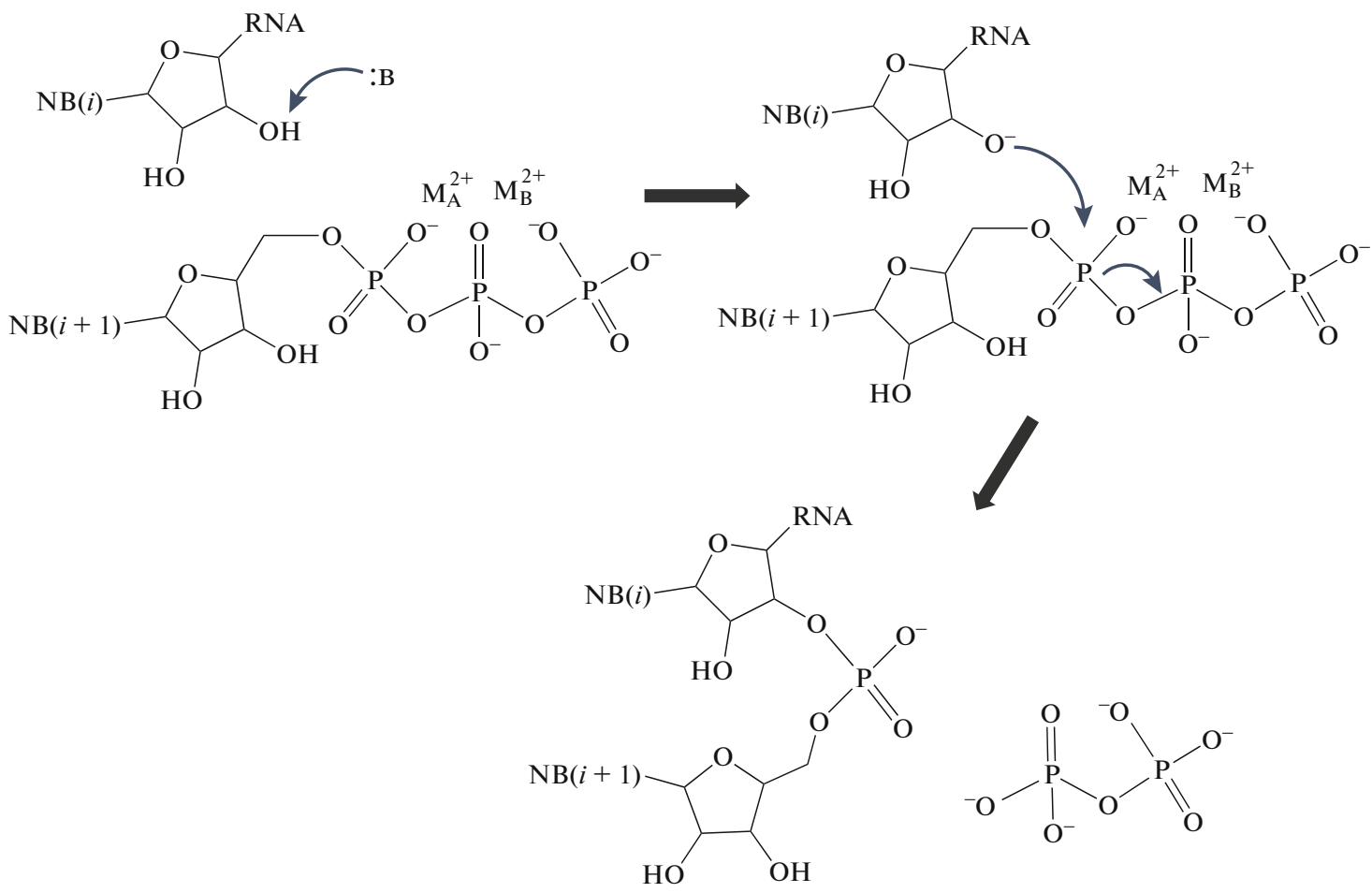

Fig. 1. The mechanism of chemical transformations in the active center of RNA polymerase.

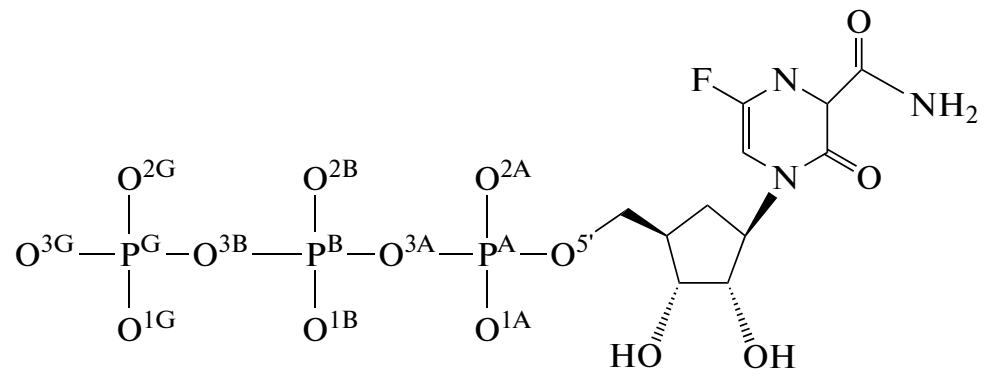

Fig. 2. Favipiravir ribosatriphosphate (FRTP) molecule.

\section{MODELS AND METHODS}

Like all coronaviruses, SARS-CoV-2 uses a multidomain molecular machine architecture to replicate and transcribe its RNA. After the cleavage of viral polyproteins, a set of unstructured proteins (nsps) is formed, three of which are included in the minimum configuration that ensures the functioning of the complex, namely, nsp12 (RdRp), nsp7, and nsp8. The work [3] reported on the structure of the nsp12nsp7-nsp8 complex without RNA strands and cofactors with a resolution of $2.9 \AA$ (PDB ID: 6M71). As an additional source of structural data, we used the results of X-ray structural analysis [4] for the RdRp enzyme of the hepatitis $\mathrm{C}$ virus. The corresponding structure of PDB ID: 4WTG, together with nsp12nsp7-nsp8, contains RNA chains, cofactors $\left(\mathrm{Mn}^{2+}\right)$, and the compound sofosbuvir (in the form of ribose diphosphate). Using the alignment procedure allowed us to compare the initial structure of the PDB ID: 6M71 and the active site of the enzyme in the structure of PDB ID: 4WTG. Further, the $\mathrm{Mn}^{2+}$ ions were replaced by $\mathrm{Mg}^{2+}$; and sofosbuvir, by FRTP (see Fig. 2); the side chains of a number of residues of the active site were rotated in accordance with the position of their analogs in the structure of PDB ID: 4WTG.

The resulting model system was completely solvated by water molecules; sodium and chlorine ions were added to neutralize the charge and ensure the physiological concentration of $\mathrm{NaCl}: 0.15 \mathrm{~mol} / \mathrm{L}$. Molecular dynamic calculations were carried out using the NAMD program [8]; and trajectory analysis, using the VMD program [9]. The conditions of the 


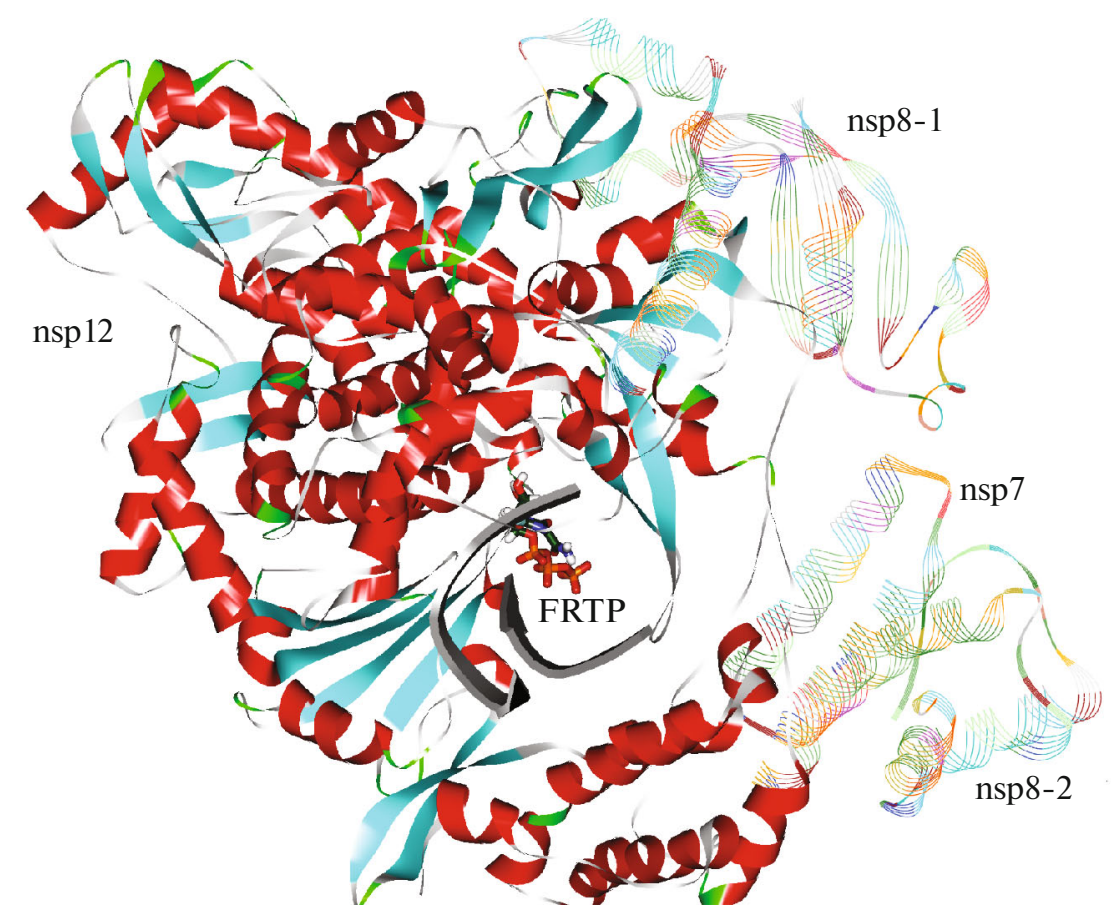

Fig. 3. General view of the model system without solvation shells.

NTP ensemble with periodic boundary conditions were used; the integration step was $2 \mathrm{fs}$, and all bonds of heavy atoms with hydrogen atoms were fixed (SHAKE/SETTLE algorithms). The interaction's potentials corresponded to the CHARMM36 force field [10]. The custom topology and parameters of the FRTP remainder were described by the CGenFF parameters [11]. The length of the trajectories was at least $40 \mathrm{~ns}$, and the trajectory frames were recorded in intervals of 200 ps.

\section{RESULTS AND DISCUSSION}

Figure 3 shows a complex without solvating shells of water molecules. The nsp7 and nsp8 proteins are important for maintaining the structure but are not involved in chemical transformations.

The center of Fig. 3 shows the phosphate groups of the FRTP molecule shown by the rods. The arrows indicate the RNA chains containing guanine (Gua), adenine (Ade), and uracil (Ura) nucleotides.

Figure 4 shows how stable the model system is along the molecular dynamic trajectory. After the initial section (about $10 \mathrm{~ns}$ ), the whole structure is quite stable (Fig. 4a). The analysis shows that the most mobile regions in nsp 12 belong to loops far from the active site of the enzyme.

It takes more time to equilibrate the FRTP position-approximately $20 \mathrm{~ns}$. This is explained by the fact that the FRTP molecule is not a native substrate of the enzyme and by the features of the initial stage of constructing the model system: FRTP groups replaced another compound in the crystal structure of PDB ID: 4WTG.

Figure 5 shows that the favipiravir molecule is quite successfully incorporated into the complex. The FRTP "head" is structurally similar to adenine and pairs with the nearest base, Gua. Figure 6 shows the structure of the active site of the RdRp enzyme.

The favipiravir is attached to the terminal Ura nucleotide according to the chemical reaction shown in Fig. 1. At the first stage, the $\mathrm{O}^{3 \prime} \mathrm{H}^{3 \prime}$ ribose group is deprotonated of uracil, and the obvious generalized base is the side chain Asp760-the distance between the oxygen atom $\mathrm{O}^{3}$, and the oxygen atom of the carboxyl group of aspartic acid is $2.8 \AA$, providing efficient proton transfer. The distance from $\mathrm{O}^{3 \prime}$ to the phosphorus atom $\mathrm{P}^{\mathrm{A}}$, equal to $3.3 \AA$, also favors nucleophilic attack with the formation of the $\mathrm{O}^{3 \prime}-\mathrm{P}^{\mathrm{A}}$ bond and breaking of the $\mathrm{P}^{\mathrm{A}}-\mathrm{O}^{3 \mathrm{~A}}$ bond. Figure 6 does not show the side chains of the amino acid residues Asp761 and Asp618 included in the coordination spheres of the magnesium ions or the positively charged residues Arg553, Arg555, and Lys551, which bind and orient the triphosphate site of the FRTP molecule.

This study confirms the modern achievements of computer modeling of biomolecular processes [12, 13]. In this case, the constructed active center of the enzyme is fully consistent with the concept of catalytic 

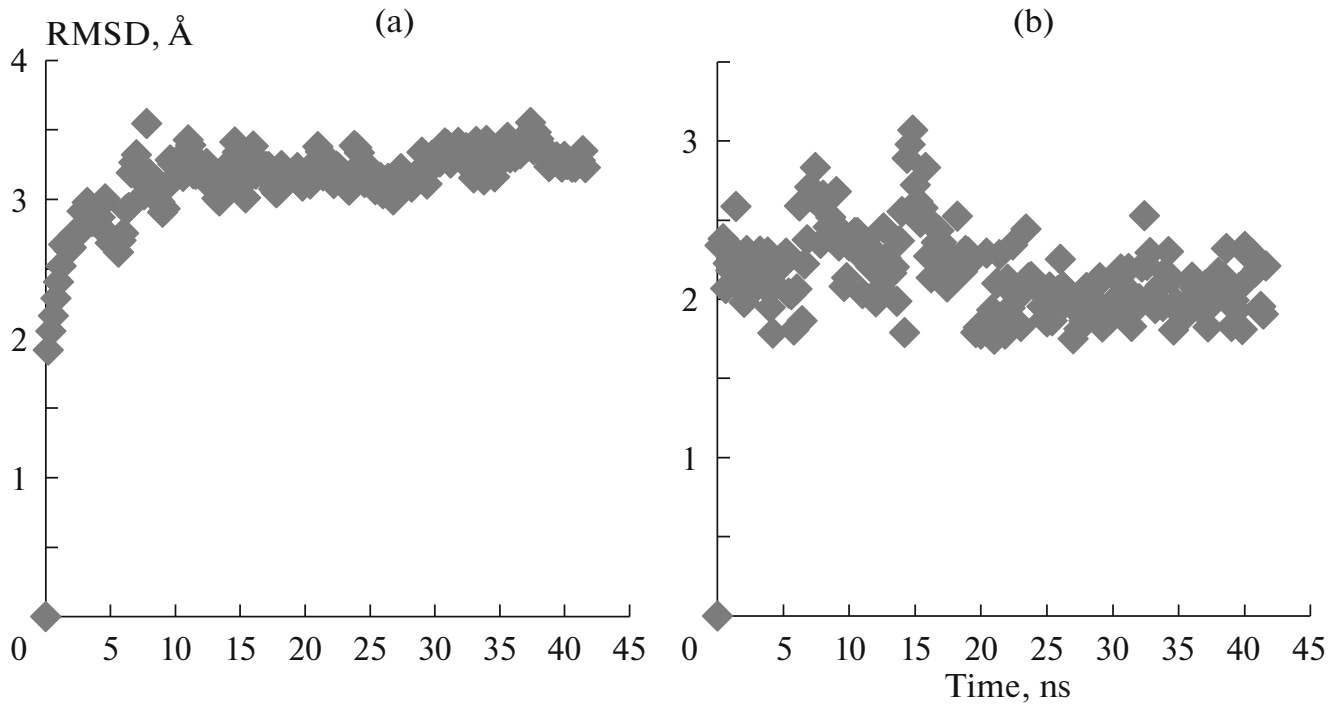

Fig. 4. Root mean square deviations (RMSD) of heavy protein atoms (a) and FRTP molecules (b).

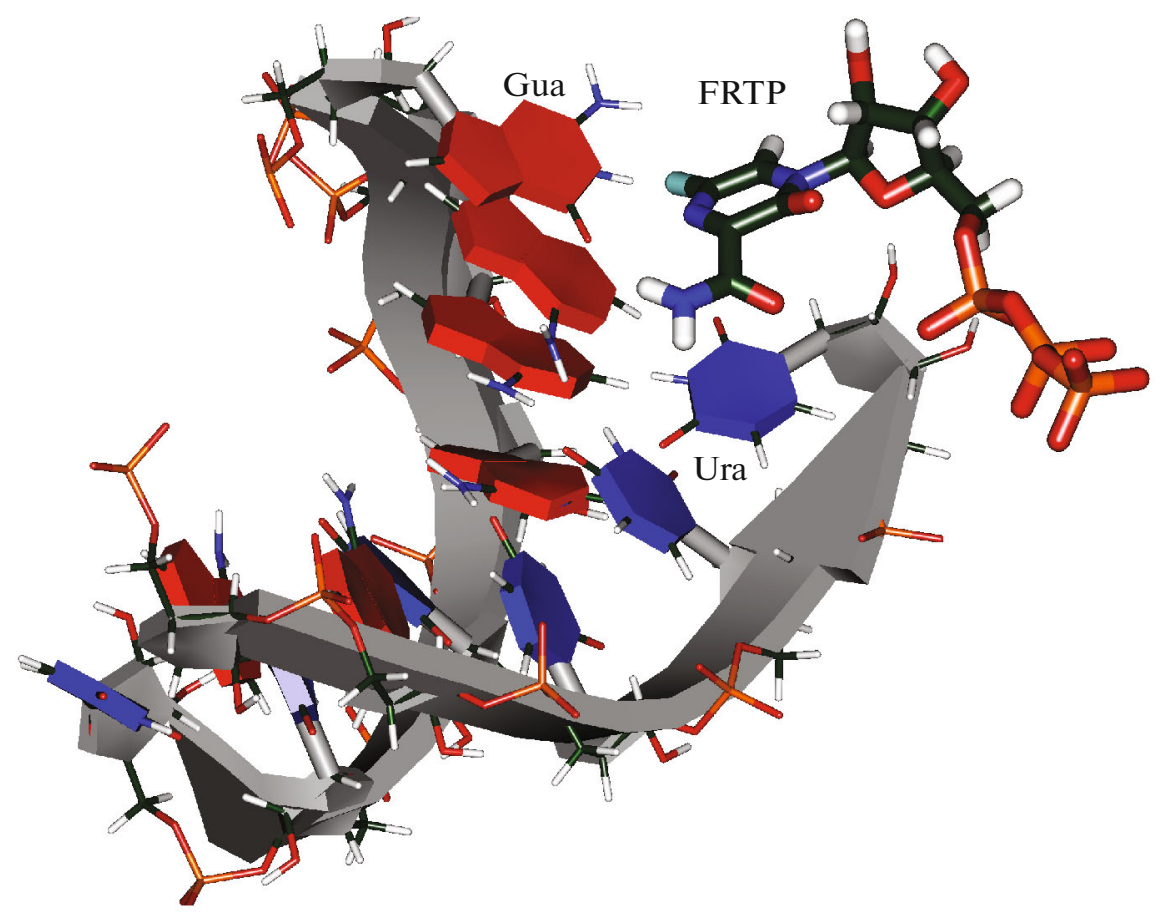

Fig. 5. RNA chains and favipiravir in a complex.

processes involving nucleoside triphosphates. A similar active site is formed in the case of the enzyme adenylate cyclase, which catalyzes the conversion of adenosine triphosphate to cyclic adenosine monophosphate. The elementary stages of this process also include deprotonation of the $\mathrm{O}^{3 \prime} \mathrm{H}^{3 \prime}$ groups of ribose, nucleophilic attack of $\mathrm{O}^{3}$, on the phosphorus atom $\mathrm{P}^{\mathrm{A}}$ of the $\alpha$-phosphate group of nucleoside triphosphate and cleavage of the $\mathrm{P}^{\mathrm{A}}-\mathrm{O}^{3 \mathrm{~A}}$ bond. The recent calculations using the combined method of quantum mechanics/molecular mechanics [6] showed that the energy profile of this reaction has a barrier for the limiting stage of the order of $16 \mathrm{kcal} / \mathrm{mol}$. We expect that in the case of the reaction of SARS-CoV-2 RNA polymerase with FRTP, the energy profile will be similar, which is consistent with the efficient incorporation of favipiravir in the RNA chain. The blockade of RNA 


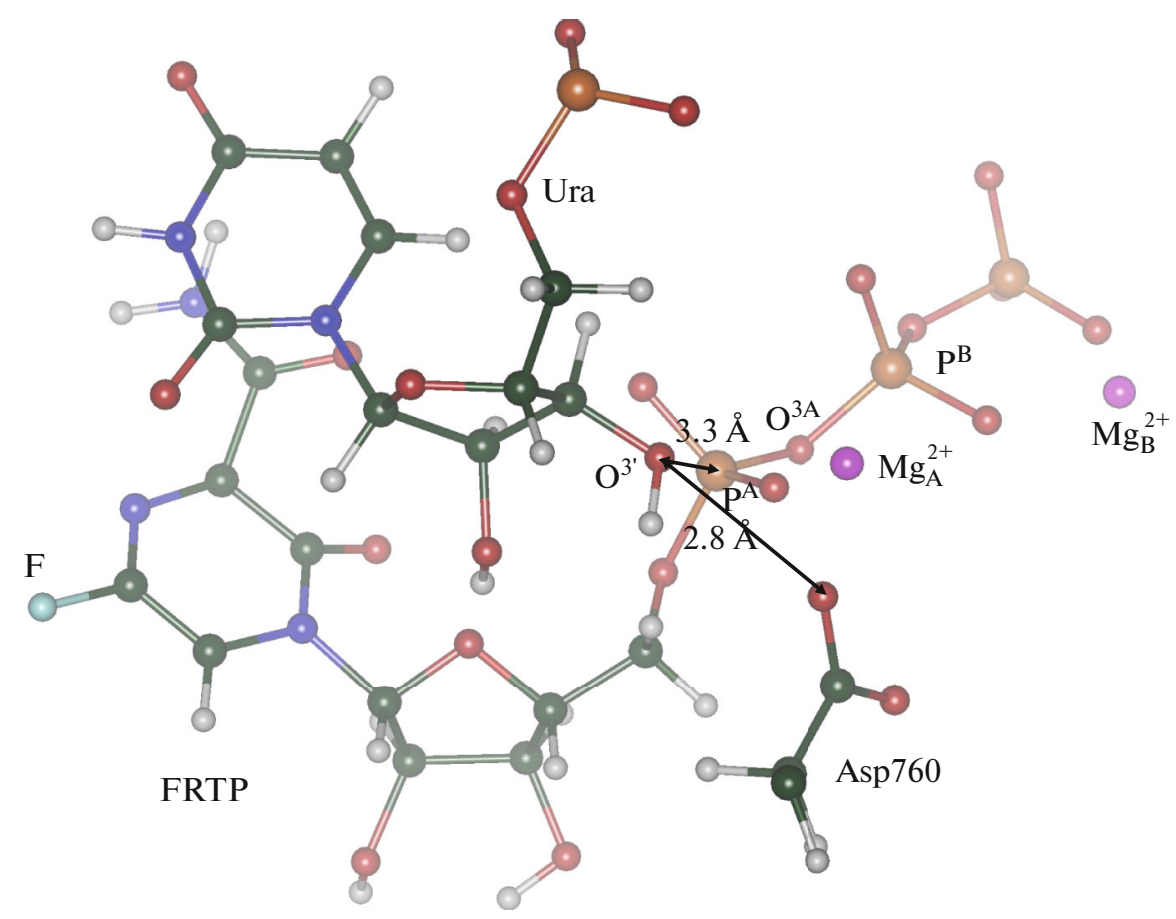

Fig. 6. Molecular groups in the active site of RNA polymerase with favipiravir.

synthesis after the capture of this compound is most likely related to a violation of the structure of the forming chain.

\section{CONCLUSIONS}

A model of the multidomain RNA polymerase complex of the SARS-CoV-2 coronavirus constructed by molecular modeling methods explains the mechanism of favipiravir's effect on the replication process. The analysis of the obtained structure and the active center of the enzyme shows that favipiravir in the form of ribosatriphosphate can integrate into the nsp12-nsp7-nsp8 complex with RNA chains and compete with the natural nucleotides in the chain extension reaction.

\section{FUNDING}

This study was financially supported by the Russian Foundation for Basic Research (project no. 19-03-00076) using the equipment of the Interdepartmental Supercomputer Center of the Russian Academy of Sciences and the Center for Shared Use of Ultra High Performance Computing Resources of Moscow State University.

\section{REFERENCES}

1. S. Venkataraman, B. V. L. S. Prasad, and R. Selvarajan, Viruses 10, 76 (2018). https://doi.org/10.3390/v10020076

2. Y. Furuta, B. B. Gowen, K. Takanashi, et al., Antiviral Res. 100, 446 (2013).

https://doi.org/10.1016/j.antiviral.2013.09.015
3. Y. Gao, L. Yan, Y. Huang, et al., Science (Washington, DC, U. S.) 368, 779 (2020). https://doi.org/10.1126/science.abb7498

4. T. C. Appleby, J. K. Perry, E. Murakami, et al., Science (Washington, DC, U. S.) 347, 771 (2013). https://doi.org/10.1126/science.1259210

5. B. Wang, M. Feig, R. I. Cukier, et al., Chem. Rev. 113, 8546 (2013). https://doi.org/10.1021/cr400046x

6. B. Grigorenko, I. Ployakov, and A. Nemukhin, J. Phys. Chem. B 124, 451 (2019). https://doi.org/1021/acs.jpcb.9b07349

7. B. L. Grigorenko, E. D. Kots, and A. V. Nemukhin, Org. Biol. Chem. 17, 4879 (2019). https://doi.org/10.1039/c9ob00463g

8. J. C. Phillips, R. Braun, W. Wang, et al., J. Comput. Chem. 26, 1781 (2005). https://doi.org/10.1002/jcc.20289

9. W. Humphrey, A. Dalke, and K. Schulten, J. Mol. Graph. 14, 33 (1996). https://doi.org/1016/0263-7855(96)00018-5

10. R. B. Best, X. Zhu, J. Shim, et al., J. Chem. Theory Comput. 8, 3257 (2012). https://doi.org/10.1021/ct300400x

11. K. Vanommeslaeghe, E. Hatcher, C. Acharya, et al., J. Comput. Chem. 31, 671 (2010). https://doi.org/10.1002/jcc.21367

12. B. L. Grigorenko, M. G. Khrenova, A. M. Kulakova, and A. V. Nemukhin, Russ. J. Phys. Chem. B 14, 457 (2020). https://doi.org/10.31857/S0207401X20060023

13. Yu. I. Meteleshko, A. V. Nemukhin, and M. G. Khrenova, Russ. J. Phys. Chem. B 13, 389 (2019).

https://doi.org/10.1134/S0207401X19060074 